Revista Científica General José María Córdova, Bogotá, Colombia, enero-junio, 2017

Recensión - Vol. 15, Núm. 19, pp. 349-362

ISSN 1900-6586

Cómo citar este artículo: Ortiz, A. (2017, enero-julio). La nueva ciencia que visiona Fritjof Capra. Rev. Cient. Gen. José María Córdova 15(19), 349-362. DOI: http://dx.doi.org/10.21830/19006586.89

\title{
La nueva ciencia que visiona Fritjof Capra*
}

DOI: http://dx.doi.org/10.21830/19006586.89

Recibido: 4 de septiembre de 2016• Aceptado: 2 de noviembre de 2016

The new science that envisions Fritjof Capra

La nouvelle science qui envisage de Fritjof Сapra

A nova ciência que prevê Fritjof Capra

Alexander Ortiz Ocaña ${ }^{a}$

* Artículo de reflexión derivado del proyecto de investigación "Competencias laborales sobre TIC en las pymes colombianas" (Grupo de Investigación Umbrales, Universidad Cooperativa de Colombia, Santa Marta).

a Universidad Cooperativa de Colombia, Santa Marta, Colombia. Doctor en Ciencias Pedagógicas, Universidad Pedagógica de Holguín, Cuba. Doctor Honoris Causa en Iberoamérica, Consejo Iberoamericano en Honor a la Calidad Educativa (CIHCE), Lima. Perú. Magíster en Gestión Educativa en Iberoamérica, CIHCE, Lima, Perú. Magíster en Pedagogía Profesional, Universidad Pedagógica y Tecnológica de La Habana. Contador Público. Recibió el premio a la excelencia educativa 2007 y 2008 otorgado por el CIHCE con sede en Lima, Perú. Mejor pedagogo novel de Cuba en el año 2002. Ha realizado asesorías pedagógicas, talleres y conferencias en Cuba, México, Brasil, Ecuador, Venezuela, Chile, Paraguay y Panamá, así como en múltiples instituciones educativas y universidades de Colombia. Docente de la Facultad de Ciencias Administrativas, Contables y de Comercio Internacional de la Universidad Cooperativa de Colombia, Santa Marta. <alexanderortiz2009@gmail.com> 
Resumen. Durante el desarrollo del proyecto de investigación "Competencias laborales sobre TIC en las pymes colombianas", desarrollado por el Grupo de Investigación Umbrales, de la Universidad Cooperativa de Colombia, Santa Marta, Colombia, luego de analizar las implicaciones de las tecnologías de la información y las comunicaciones en las competencias laborales, y de analizar distintas clasificaciones sobre las TIC y sobre competencias laborales, constatamos que existen otras propuestas investigativas holísticas y ecológicas, derivadas de nuevas epistemologías. A partir de aquí surge la necesidad de escribir y publicar este artículo, en el que se hace una reflexión acerca de la nueva ciencia que visiona Fritjof Capra. Se analiza la nueva física como cimiento y fundamento epistemológico de la nueva ciencia. Se postula al organicismo desde la biología como germen de la Teoría General de Sistemas y, por último, se reflexiona acerca de la psicología de la Gestalt y la fenomenología.

Palabras clave: nueva ciencia, epistemología, organicismo, Teoría General de Sistemas, psicología de la Gestalt, fenomenología.

Abstract. During the development of the research project work skills on ICT in the Colombian SMEs, developed by the research group Umbrales, of the University Cooperative of Colombia, Santa Marta, Colombia, after analyzing the implications of the technologies of the information and communications skills, and analyzing different classifications on ICT and competency, we see that there are other proposed teaching holistic and ecological, derived from new epistemologies, and from here arises the need to write and publish this article, which is a reflection on the new science Fritjof Capra to view. Discusses the new physics as the Foundation and epistemological Foundation of the new science. It is postulated to organicism from biology such as germ of general systems theory and finally reflects about Gestalt psychology and phenomenology.

Keywords: new science, epistemology, organicism, general theory of systems, Gestalt psychology, phenomenology.

Résumé. Au cours du développement des compétences de travail de projet de recherche sur les TIC dans les PME colombiennes, mis au point par Umbrales de groupe de recherche, de la coopérative Université de la Colombie, Santa Marta (Colombie), après avoir analyser les incidences des technologie et des communications personnelles et analysé les différentes classifications sur les TIC et les compétences Nous pouvons voir qu'il existe un autre projet d' investigation holistique et écologique, dérivée de nouvelles épistémologies et de là découle la nécessité d'écrire et de publier cet article, qui devient une réflexion sur la nouvelle science qui envisage de Fritjof Capra. Traite de la nouvelle physique, comme la Fondation et le fondement épistémologique de la science nouvelle. Ile st postulé que l'organicisme de biologie comme le germe de la théorie générale des systèmes et enfin réfléchit sur la psychologie de la Gestalt et la phénoménologie.

Mots-clés : nouvelle science, l'épistémologie, l'organicisme, théorie générale des systèmes, Gestalt théorie, phénoménologie.

Resumo. Durante o desenvolvimento das habilidades de trabalho de projeto de pesquisa sobre as TIC nas PME colombiano, desenvolvido pelos Umbrales de grupo de pesquisa, da Universidade cooperativa da Colômbia, Santa Marta, Colômbia, depois de analisar as implicaçôes das habilidades de tecnologia e comunicação de informaçóes e analisar diferentes classificaçôes em TIC e competência Podemos ver que existem outras propostas investigativo holística e ecológica, derivado de novas epistemologias e daqui resulta a necessidade de escrever e publicar este artigo, no qual torna-se uma reflexão sobre a nova ciência que visiona Fritjof Capra. Discute a nova física como a Fundação e a Fundação epistemológica da nova ciência. Ele é postulado como organicismo de biologia como germe da teoria geral dos sistemas e finalmente reflete sobre a psicologia da Gestalt e da fenomenologia.

Palavras-chave: nova a ciência, epistemologia, organicismo, teoria geral de sistemas, psicologia da Gestalt, fenomenologia. 


\section{Introducción}

En los últimos años del siglo $X X$, y en la primera década del presente siglo $X X I$, estamos asistiendo y participando de notables cambios en la epistemología, que han acompañado y se han nutrido de la inminente transformación paradigmática en las ciencias. Sin lugar a dudas estamos ad portas de una inigualable revolución científica, una profunda metamorfosis de las concepciones epistemológicas. En realidad el problema es más profundo, se trata de una crisis del pensamiento. De ahí que se avecina o emerge con fuerza una transformación en la filosofía de la ciencia que ofrecerá nuevas visiones de la realidad y nuevas formas de hacer ciencia. De esta manera, nuevas categorías han ido dando forma a nuestra visión sobre el mundo que nos rodea, entre las cuales se destaca la noción de "red", que hoy ocupa un lugar significativo en la configuración de sentido y significado, tanto en las ciencias fácticas o naturales (mal llamadas ciencias "duras") como en las ciencias sociales o humanas, o del espíritu, como diría Dilthey (1951).

Pensar en red significa e implica la posibilidad de tener en cuenta el alto grado de relación, interconexión y configuración de los fenómenos, eventos y procesos, y establecer ámbitos de conocimiento sustentados en las diversas formas del conocimiento y la experiencia humana y sus múltiples articulaciones.

El ser humano es un artista que a su vez integra su propio arte, por lo cual configura el mundo y le da sentido y significado a este y, a su vez, él mismo va reconfigurándose en las múltiples interconexiones que tiene con los demás seres humanos, con él mismo y con los procesos, eventos, fenómenos, acontecimientos y situaciones con los que interactúa.

El siglo $X X I$ avanza a pasos agigantados guiado por la complejidad y las nuevas teorías de sistemas, tanto en la ciencia como en la epistemología. La ciencia clásica se caracterizó por privilegiar el determinismo, la linealidad, el reduccionismo, la predicción, la causalidad y la conservación mecanicista, dogmática y esquemática, pero los nuevos paradigmas han puesto su mirada en los procesos complejos, sistémicos, dinámicos, no lineales y relacionales.

En los albores del siglo XXI la modernidad acelera nuestra aversión hacia ella debido a sus inertes paradigmas que aún dominan en el mundo científico de Occidente desde sus silenciosas tumbas, con pueriles discursos que demuestran su inoperancia. Estos paradigmas hoy son muertos insepultos. Es por ello que estamos buscando opciones y enfoques más pertinentes, viables y sostenibles, un momento de configuración de nuevos paradigmas, más tolerantes, incluyentes y comprensivos. En este sentido, son destacables, tienen sentido y pertinencia los aportes de Fritjof Capra, reconocido y prestigioso físico austriaco, nacido el 1..$^{\circ}$ de febrero de 1939, en Viena.

A los 27 años Capra culminó un doctorado en Física teórica, en la Universidad de Viena. Luego fue profesor en la Universidad de San Francisco, en Berkeley, y en la Universidad de California (U. C.), en Santa Cruz. También se desempeñó haciendo investigaciones en física subatómica en el Laboratorio Lawrence Berkeley de la U. C., en el Acelerador Lineal de Londres y en la Universidad de París.

Fritjof Capra es un consagrado experto en la nueva teoría de sistemas, rama de investigación en la que incursionó después en la década de los 80 , después de haber dedicado más de 20 años a la física de partículas. 
Su producción intelectual (Capra, 1988, 1991, 2003, 2007, 2008a, 2008b, 2009, 2010), cuestionable, polémica y controvertida, ha influido de manera considerable en la comunidad científica internacional y ha contribuido significativamente en la formulación del nuevo paradigma holístico y ecológico, sobre todo a partir de una de sus obras más importantes: El punto crucial (1982).

Simultáneamente a estas actividades docentes y científicas, Capra ha estudiado de forma profunda durante más de 40 ańos las consecuencias de la ciencia clásica en el pensamiento occidental moderno, tanto desde el punto de vista filosófico como social. Sobre este tema ha publicado innumerables libros y artículos científicos, y frecuentemente hace conferencias y seminarios en diversos países. Sus obras se basan en la interconexión entre todos los fenómenos del universo, su teoría de la convergencia entre física y metafísica y las relaciones entre el misticismo antiguo, fundamentalmente el oriental, y el universo develado por la física moderna. Precisamente, El tao de la física (1975) fue el best-seller que se convirtió en la génesis de múltiples obras sobre este tema. Este ícono moderno marcó un hito importante en la producción intelectual y científica de este prestigioso físico teórico.

Otro notable físico, Werner Heisenberg, estremeció la palestra científica internacional que defendía la predictibilidad de la naturaleza, mediante axiomas infalibles, teoremas y ecuaciones. Esto lo logró con su controversial enunciación del principio de la indeterminación. Capra reconoció años más tarde el gran aporte que había recibido su obra de las formulaciones de Heisenberg. En efecto, la obra de Capra evidenció la impresionante similitud entre las tradiciones espirituales de Oriente y las teorías científicas aceptadas en ese momento, lo cual ya había sido reconocido décadas antes de manera privada por diversos científicos e intelectuales de la época.

Desde los años ochenta, Capra se dio cuenta de que a lo largo de quince años había seguido persistentemente un solo tema: el cambio esencial de la cosmovisión que estaba sucediendo en la sociedad y en la ciencia, así como la aparición, desarrollo y consolidación de una nueva mirada acerca de la realidad y las inminentes consecuencias sociales de dicha transformación cultural.

Del crisol de estos nuevos paradigmas surge la psicología transpersonal como nueva disciplina que estudia los procesos psíquicos humanos desde una mirada más holística y ecológica. El padre de esta disciplina es Stanislav Grof (s. f.), quien en uno de sus escritos expresó:

Por lo general no se menciona en la mayoría de los libros de texto que muchos de los fundadores de la física moderna, tales como Einstein, Bohm, Heisenberg, Schrödinger, Bohr y Oppenheimer, no solo hallaron su trabajo plenamente compatible con la visión mística del mundo, sino que en cierto modo entraron en el campo místico a través de la investigación científica (p. 31).

En efecto, al profundizar en la obra de estos eminentes científicos se percibe que las miradas de las tradiciones ancestrales espirituales articulan de manera armónica y coherente con las miradas de la naturaleza centradas en el papel esencial del observador en la percepción de los fenómenos naturales.

Por un lado, Stanislav Grof, a partir de su residencia en el Instituto Esalen, afirmó que la ciencia moderna es netamente neopitagórica y neoplatónica, influido por el eminente ecólogo británico Gregory Bateson (1904-1980), con quien compartió estas visiones neosistémicas, ho- 
lísticas y ecológicas. Esto significa que la visión de Pitágoras, Platón y la nueva ciencia pueden ser fundamentadas desde el reconocimiento de estas tradiciones místicas.

Por otro lado, los trabajos de investigación y divulgación de Capra, posteriores a la circulación de su controvertido libro El tao de la física, incluyen estudios sobre otros campos científicos diferentes a la física, como la ecología, la biología y la psicología, inspirado también en la obra de Bateson (2010, 2011). En su primer libro, Capra plantea unos postulados interesantes y controversiales, en los cuales enfatiza la importancia de analizar nuestro universo desde una mirada holística y de lograr una comprensión ecológica de este, relacionándolo con la trama de la vida, a partir de su pertenencia a la infinita y extensa danza de la creación. Esto demuestra que su concepción ontológica se sustenta en una visión sobre la naturaleza de la realidad como un proceso creativo e interconectado en el que su comprensión implica un análisis holístico, no aislado.

Los propios esfuerzos de Capra han estado encaminados precisamente a cambiar la ciencia actual, de tal modo que dichas consideraciones pudieran incorporarse en el marco científico y epistemológico del futuro. Para ello, hace hincapié en que el primer paso debía consistir en cambiar el enfoque mecanicista y fragmentario de la ciencia clásica por un paradigma holístico y ecológico, en el cual el énfasis principal se desplazara de las entidades independientes a las relaciones. Esto permitiría introducir las categorías de contexto y significado. Capra afirma que solo cuando se dispusiera de dicho marco eco-holista se podrían empezar a dar los pasos necesarios en respuesta a las preocupaciones de una gran diversidad de científicos. Y él logra hacerlo de manera excepcional, sustentado en los postulados de Laing (1978, 1983), Laszlo (1989) y Prigogine (2008, 2009, 2012).

En correspondencia con lo anterior, Capra (2009) considera que "nuestras instituciones sociales son incapaces de resolver los principales problemas de nuestra época, por el hecho de aferrarse a una visión anticuada del mundo, la visión mecanicista de la ciencia del siglo XVIP' (p. 254). El modelo clásico de la física newtoniana y el método cartesiano han servido de cimiento epistemológico y metodológico no solo para las ciencias naturales, sino también para las ciencias humanas y sociales, y las limitaciones de esta visión del mundo son ahora evidentes en múltiples aspectos de la crisis global. Si bien el modelo newtoniano es todavía el paradigma dominante en nuestras instituciones académicas y en la sociedad en general, los físicos han ido mucho más allá.

Capra afirma que de la nueva física emerge una nueva cosmovisión, que describe con sus énfasis en la interconexión, las relaciones, la trama, las pautas dinámicas, la red, y los cambios y las transformaciones permanentes, lo cual generará en otras ciencias un cambio de su filosofía subyacente, con el fin de ser consecuentes con esta nueva visión de la realidad, cuyo cambio radical, según Capra (2009), “constituye el único modo de resolver verdaderamente nuestros urgentes problemas económicos, sociales y ambientales” (p. 255).

Los biólogos moleculares no comprendieron los procesos interconectores holísticos de los sistemas vivos, a pesar de que descubrieron los componentes básicos de la vida. Hace más de veinte años, Sydney Brenner, uno de los principales biólogos moleculares, afirmaba:

De algún modo, podríamos decir que todo el trabajo realizado en los campos de la genética y de la biología molecular de los últimos sesenta años, podría ser considerado como un largo intervalo [...]. Ahora que el programa ha sido completado, nos encontramos de nuevo con los problemas que se 
dejaron sin resolver. ¿Cómo se regenera un organismo dañado hasta recuperar exactamente la misma estructura que tenía antes de producirse el daño? ¿Cómo forma el huevo al organismo? [...] (Citado en Capra, 2010, p. 19).

A partir de estas consideraciones, los científicos configuraron un nuevo lenguaje para el estudio de los organismos vivos, dada su complejidad y alto nivel de integración e interconexión: sistemas complejos, dinámica de redes, dinámica no-lineal, teoría de la complejidad, teoría de los sistemas dinámicos, configuraciones vivas, psíquicas y sociales, etc. De manera que algunos de los conceptos clave de estas nuevas teorías sistémicas son: autoorganización, estructuras disipativas, fractales, atractores caóticos, redes autopoiéticas y configuración.

Sin embargo, a pesar de lo anterior, el pesimismo se ha apoderado de la voluntad de los científicos. Sin lugar a dudas, existen soluciones muy sencillas para los problemas fundamentales de nuestra época, pero para ello se necesita una inmensa transformación en nuestro discernimiento, en nuestra forma de ver el mundo, en nuestro pensamiento, en nuestra concepción de ciencia, del conocimiento, en nuestros valores, en la forma de analizar la esencia y naturaleza de los objetos de estudio (ontología) y, por supuesto, del proceso o lógica de configuración del conocimiento científico (epistemología). Sin duda, está emergiendo esta transformación esencial de mirada en la sociedad y en la actividad científica, el surgimiento de nuevos paradigmas científicos, tan radicales o más impactantes y trascendentales que la revolución que provocó Copérnico.

\section{La nueva física como cimiento y fundamento epistemológico de la nueva ciencia}

$\mathrm{Al}$ explorar la naturaleza de los fenómenos subatómicos y la estructura de los átomos, los más grandes y eminentes físicos del siglo $X X$ quedaron perplejos. Así lo han descrito los fundadores de la teoría cuántica, entre ellos Heisenberg, pero también Albert Einstein y Niels Bohr, uno de los gigantes de la física moderna, quienes escriben y analizan el singular dilema que percibieron los físicos que cambiaron de manera radical su forma de pensar, y así se fueron destruyendo poco a poco los cimientos de su cosmovisión, por cuanto dicha exploración les hizo ver una extraña e insólita realidad que no esperaban encontrar. "El mundo material que observan había dejado de parecer una máquina, compuesta por multitud de objetos independientes, y su aspecto era más bien el de un todo indivisible; una red de relaciones que incluía de un modo esencial al observador humano" (Capra, 2009, p. 15). En sus esfuerzos por asimilar la naturaleza de los fenómenos atómicos, continúa Capra (2009), "a los científicos les dolió descubrir que sus conceptos básicos, su lenguaje y en definitiva su forma de pensar era inadecuada para expresar esa nueva realidad" (p. 16).

En Physics and Philosophy, Heisenberg nos brinda no solo un análisis brillante de los problemas conceptuales, sino también un vivo relato de las enormes dificultades personales experimentadas por dichos físicos cuando su investigación les obligó a ampliar su conciencia. "Sus experimentos atómicos les forzaron a pensar en nuevas categorías sobre la naturaleza de la realidad, y el gran acierto de Heisenberg consistió en reconocerlo claramente" (Capra, 2009, p. 16). 
Cuando Capra era un joven estudiante, leyó el libro de Heisenberg; le fascinó su relato de las paradojas y aparentes contradicciones que infestaban la investigación de los fenómenos atómicos al principio de los años veinte. Muchas de dichas paradojas estaban relacionadas con la naturaleza dual de la materia subatómica, que se presenta unas veces como partículas y otras veces como ondas.

Los físicos de aquella época solían decir que los electrones son ondas los martes y los jueves, y son partículas los lunes y los miércoles. Y lo extraño era que cuanto más se esforzaban los físicos para aclarar la situación, mayor era el contraste de las paradojas. Solo muy gradualmente desarrollaron los físicos cierta intuición en cuanto a la manifestación del electrón como partícula y como onda. Estaban adoptando, según Heisenberg, el espíritu de la teoría cuántica antes de desarrollar su formulación matemática precisa.

El propio Heisenberg tuvo un papel decisivo en dicho desarrollo. Se dio cuenta de que las paradojas en la física atómica aparecían cuando se intentaba describir los fenómenos atómicos en términos clásicos, y tuvo el valor de desechar el marco conceptual clásico. En 1925 publicó un artículo científico en el que abandonó la descripción convencional de los electrones como componentes del átomo en términos de posición y velocidad, utilizada por Bohr y por todos los demás físicos, sustituyéndola por otro marco mucho más abstracto, en el que las cantidades físicas estaban representadas por unas estructuras matemáticas denominadas matrices.

La "mecánica de las matrices" de Heisenberg fue la primera formulación con coherencia lógica de la teoría cuántica. Otra formulación distinta, elaborada por Erwin Schrödinger y conocida como "mecánica de las ondas", vino a complementarla al cabo de un año. Ambas formulaciones están dotadas de coherencia lógica y son matemáticamente equivalentes; el mismo fenómeno atómico puede ser descrito con dos lenguajes matemáticos distintos (Capra, 2009, p. 17).

A finales de 1926, los físicos disponían de una formulación matemática completa y lógicamente coherente, pero no siempre sabían cómo interpretarla para describir una situación experimental determinada. "El gran logro de Heisenberg consistió en expresar dichas limitaciones de los conceptos clásicos de una forma matemática precisa, que actualmente lleva su nombre y se conoce como principio de indeterminación de Heisenberg" (Capra, 2009, p. 18).

El principio de indeterminación mide hasta qué punto el científico influye en las propiedades de los objetos observados a través del proceso de medición. En la física atómica, los científicos ya no pueden actuar como observadores independientes y objetivos; están vinculados con el mundo que observan y el principio de Heisenberg mide dicha vinculación.

En los años veinte, encabezados por Heisenberg y Bohr, los físicos llegaron a comprender que el mundo no es una colección de objetos independientes, sino que parece más bien una red de relaciones entre las diversas partes de un todo unificado. Nuestras ideas clásicas, derivadas de la experiencia cotidiana, no son plenamente adecuadas para describir este mundo. Werner Heisenberg ha explorado — más que nadie - los límites de la imaginación humana, los límites hasta lo que cabe extender nuestros conceptos convencionales y hasta qué punto debemos vincularnos con el mundo que observamos (Capra, 2009, p. 19).

En criterio de Capra (2009), el hecho de que todos los conceptos y teorías de la ciencia son aproximaciones a la auténtica naturaleza de la realidad, válidos solo para cierta gama de fenómenos, 
fue evidente para los físicos a principios de este siglo, gracias a los espectaculares descubrimientos que conllevaron la configuración de la teoría cuántica. Desde entonces, "los físicos han aprendido a ver la evolución del conocimiento científico en términos de una secuencia de teorías, o modelos, cada uno más preciso y de mayor alcance que el anterior, pero sin que ninguno de ellos represente una versión completa y definitiva de los fenómenos de la naturaleza” (p. 78).

Chew, por ejemplo, considera que la ciencia del futuro tendría un estilo "bootstrap”, estaría conformada por modelos entrelazados, sería un mosaico de teorías, en el que ninguna de ellas sería más importante que la otra y todas ellas estarían entrelazadas para formar un todo único consistente. Es evidente que lo que Chew está proponiendo es una ciencia total, holística y configuracional.

Nuestras instituciones sociales son incapaces de resolver los principales problemas de nuestra época, por el hecho de aferrarse a una visión anticuada del mundo, la visión mecanicista de la ciencia del siglo XVII. Tanto las ciencias naturales como las sociales y las humanidades se han ajustado al modelo clásico de la física newtoniana, y las limitaciones de esta visión del mundo son ahora evidentes en múltiples aspectos de la crisis global. Si bien el modelo newtoniano es todavía el paradigma dominante en nuestras instituciones académicas y en la sociedad en general [...] los físicos han ido mucho más allá (Capra, 2009, p. 254).

Capra describe la visión del mundo que ve emerger de la nueva física, con sus énfasis en la interconexión, las relaciones, las pautas dinámicas y los cambios y transformaciones permanentes, y expresa su convicción de que otras ciencias deberían cambiar su filosofía subyacente en consonancia, a fin de ser consecuentes con esta nueva visión de la realidad.

Hemos de ser muy cautelosos cuando decidimos que esto causa aquello y aquello causa esto, porque solemos pasar por alto muchos factores de gran importancia. Los factores genéticos tampoco tienen una importancia desmesurada. Tenemos tendencia a tratar la genética como si fuese una especie de magia (Capra, 2009, p. 322).

Debemos cambiar radicalmente nuestra forma de pensar reduccionista y determinista hacia una más sistémica, holística y configuracional. Este cambio radical constituye el único modo de resolver verdaderamente nuestros urgentes problemas económicos, sociales y ambientales.

Tengo la firme convicción de que la ciencia del tercer milenio, la Configuralogía, será capaz de ocuparse de la totalidad de la gama de los fenómenos naturales, personales y socio-culturales de un modo unificado y holístico, utilizando nuevas nociones y conceptos distintos pero consecuentes, congruentes, armónicos y coherentes entre sí para explicar diferentes fenómenos del entorno natural y socio-humano. La teoría emergente de los sistemas vivos y autoorganizadores, que se aproxima bastante a una descripción holística de la memoria, la mente y la vida, confirma mis convicciones, que han sido enriquecidas con los invaluables aportes de las nuevas teorías de sistemas.

Los biólogos han ido llegando a concepciones similares que los conducen a considerar al organismo como su categoría científica fundamental y su principal unidad de análisis en las investigaciones. De esta manera, el organicismo desde la biología se convierte en el germen de la Teoría General de Sistemas. 


\section{El organicismo desde la biología como germen de la Teoría General de Sistemas}

El organicismo es otro modelo epistémico derivado del biologicismo. El modelo organicista analiza al organismo como un cuerpo conformado por instrumentos. El representante fundamental fue Tomás de Aquino, para quien todo el organismo obedece a leyes biológicas y a las condiciones orgánicas.

El desplazamiento del pensamiento mecanicista al sistémico estuvo dado por el cambio de función a organización, por cuanto función es un concepto básicamente mecanicista. La antigua noción de función en fisiología había sido reemplazada de manera gradual por el concepto de organización, que había sido explorado por otro de los fundadores de la escuela organicista: Ross Harrison, quien "identificaba configuración y relación como dos aspectos de la organización, unificados subsiguientemente en el concepto de patrón o pauta como la configuración de relaciones ordenadas" (Capra, 2010, p. 46).

Ludwig von Bertalanffy $(1976,1978,2007)$ en el campo de la biología supera el pensamiento analítico-sumativo que era considerado el método fundamental de esta ciencia, en el cual el organismo como conglomerado actúa o reacciona solo ante la acción de factores externos en forma reflectiva, revelando los vínculos existentes entre los principios de la biología teórica y la teoría de sistemas, al emplear el método sistémico, que reconoce que el sistema está dotado de una estructura dinámica que lo hace activo en su interior.

Bertalanffy promueve la noción de sistema abierto en la biología en 1932, al tener en cuenta la relación de intercambio que se genera entre el organismo vivo y su entorno en tres aspectos: energía, sustancia e información, con lo cual aporta el eslabón que permitía unir las teorías de sistemas y las biológicas. Aunque inicialmente esto solo fue considerado en los sistemas biológicos, también fue llevado a la física y, en particular, al estudio de sistemas termodinámicos y a la cinética física.

La Teoría General de Sistemas ha hecho más énfasis en el análisis estructural de carácter sincrónico que en el diacrónico, lo que no contribuye a la interpretación histórico social, por lo cual, si bien se acerca a una interpretación dialéctica al modelar la realidad objetiva, como formada por sistemas de elementos componentes relacionados unos con otros y con una estructura compleja, se aleja de ella, al no adentrarse en el proceso del desarrollo y evolución histórica de esos sistemas.

En correspondencia con lo anterior, se pueden derivar como limitantes fundamentales en el uso de la concepción sistémico estructural para el empleo de la interpretación del proceso de pensamiento, los aspectos siguientes:

- La reducción al enfoque sincrónico, que no permite revelar la historicidad del proceso mental.

- La aplicación de un enfoque mecanicista y por tanto unilateral, que hace reducir el estudio de los elementos componentes del sistema cognitivo a la suma de las partes que lo integran.

- Una concepción limitada del modelo de la totalidad desde una mirada metodológica en el proceso de comprensión de la realidad cognitiva, que implicaría una visión también 
insuficiente del pensamiento que no permitirá interpretar el proceso mental con la requerida profundidad.

Como se aprecia, la teoría de los sistemas establece un cimiento epistemológico importante para un pensamiento de la organización, a pesar de sus evidentes limitaciones. Sin lugar a dudas, el todo es más que la suma de las partes, por cuanto existen cualidades y atributos que emergen, o sea, que se generan a partir de la organización del todo y que influyen sobre las partes, pero además, "el todo es igualmente menos que la suma de las partes, puesto que las partes pueden tener cualidades que son inhibidas por la organización del conjunto" (González, 1997, p. 17).

Tradicionalmente se reconoce a Bertalanffy como el padre y fundador de la teoría de sistemas, sin embargo, según Capra (2010) "veinte o treinta ańos antes de que publicase sus primeros escritos sobre su 'teoría general de los sistemas', Alexander Bogdanov, médico, investigador, filósofo y economista ruso, desarrolló una teoría de sistemas de igual sofisticación y alcance que, desafortunadamente, es aún muy poco conocida fuera de Rusia” (p. 63).

Tektología, del griego tekton, que significa 'constructor', es el nombre que Bogdanov le dio a su teoría, que puede ser considerada como la ciencia de las construcciones o configuraciones. La finalidad esencial de Bogdanov era sistematizar los postulados básicos que permitan analizar la esencia de organización de todas las configuraciones vivas y no vivas.

La tektología deberá clarificar los modos de organización que parecen existir en la actividad natural y humana; luego deberá generalizar y sistematizar estos modos; más adelante deberá explicarlos, es decir, proponer esquemas abstractos de sus tendencias y leyes [...] La tektología trata de las experiencias organizadoras, no de este o aquel campo especializado, sino de todos ellos en conjunto. En otras palabras, abarca la materia protagonista de todas las otras ciencias (Capra, 2010, p. 63).

Según Capra (2010), Bogdanov incluso se adelanta a las investigaciones de Ilya Prigogine (2008, 2009, 2012), y demuestra que "la crisis organizadora se manifiesta como un colapso del equilibrio sistémico existente, representando al mismo tiempo una transición organizadora hacia un nuevo estado de equilibrio" (p. 64).

Como es sabido, René Thom, eminente matemático francés, introduce la noción de catástrofe, que es esencial en las nuevas teorías de las matemáticas de la complejidad. Es evidente que Bogdanov se adelanta a este concepto de catástrofe al considerar y conceptualizar las categorías de crisis.

Según Capra (2010), el libro precursor de Bogdanov, titulado Tektología, fue publicado en tres volúmenes entre 1912 y 1917, en Rusia. Una edición alemana fue publicada y ampliamente revisada en 1928. No obstante, se conoce muy poco en Occidente sobre esta primera versión de una Teoría General de los Sistemas, precursora de la cibernética. Incluso, la Teoría General de Sistemas de Ludwig von Bertalanffy, publicada en 1968, incluye varios capítulos encaminados a describir la historia de la teoría de sistemas, sin embargo, la obra de Bogdanov no se menciona en dicha sección. Es difícil comprender cómo Bertalanffy, que publicaba todos sus trabajos originales en alemán y leía muchísimo en este idioma, no encontró la obra de Bogdanov.

Lo mismo ha ocurrido con la Teoría de las Configuraciones, desarrollada en Cuba a finales del siglo pasado para la psicología por Fernando González Rey (1997) y a inicios de este siglo 
para la pedagogía por Homero Fuentes González (2004, 2009), cuyos aportes han quedado prácticamente ocultos para los científicos de otras regiones distintas a la isla, a pesar de los desarrollos alcanzados por estas concepciones a partir de lo que yo he denominado Configuralogía o Teoría de las Configuraciones (Ortiz, 2013).

Bertalanffy creía que la Teoría General de Sistemas podría aportar un sistema de referencia conceptual con suficiente solvencia para integrar las múltiples disciplinas científicas que estaban fragmentadas y aisladas: "La teoría general de sistemas debería ser [...] un medio importante para controlar y potenciar la transferencia de principios entre campos, no siendo ya necesario duplicar o triplicar el descubrimiento del mismo principio en distintos campos aislados entre sí”. Al mismo tiempo, al formular criterios exactos, la Teoría General de Sistemas se establecería como defensa contra analogías superficiales sin utilidad para la ciencia.

Bertalanffy no llegó a ver la materialización de esta visión y quizá no haya sido nunca formulada una ciencia general de la totalidad [lugar que pretende ocupar la Configuralogía (Ortiz, 2013)]. No obstante, "durante las dos décadas siguientes a su muerte en 1972, empezó a surgir una concepción sistémica de vida, mente y consciencia que trasciende las fronteras disciplinarias y que, efectivamente, ofrece la posibilidad de unificar campos hasta ahora separados" (Capra, 2010, p. 69). Si bien esta concepción tiene sus raíces más bien en la cibernética que en la Teoría General de Sistemas, debe ciertamente mucho a las ideas y conceptos que Ludwig von Bertalanffy introdujera en la ciencia, dando lugar de esta manera al surgimiento y desarrollo del pensamiento sistémico y las nuevas teorías de sistemas, que tanta similitud tienen con los aportes de la psicología de la Gestalt.

\section{La psicología de la Gestalt}

Según Capra (2008a), la teoría gestáltica, creada por Max Wertheimer y otro inmenso grupo de psicólogos alemanes, sustenta en la idea de que "los organismos vivientes no perciben las cosas como elementos aislados sino como Gestalten, esto es, como unidades significativas dotadas de cualidades que no existen en sus partes individuales" (p. 192).

Los psicólogos Gestalt, liderados por Max Wertheimer y Wolfgang Köhler (1967), afirmaban que los seres vivos percibimos holos irreductibles. "Los organismos vivos perciben no en términos de elementos aislados, sino de patrones perceptuales integrados, conjuntos organizados dotados de significado, que exhiben cualidades ausentes en sus partes" (Capra, 2010, p. 51).

Capra (2010) nos relata que en los años veinte, tanto la biología organicista como la psicología Gestalt formaron parte de una corriente intelectual mayor que se veía a sí misma como un movimiento de protesta contra la creciente fragmentación y alienación de la naturaleza humana. "Toda la cultura Weimar se caracterizaba por su aspecto antimecanicista, por su 'hambre de totalidad' " (p. 52).

La trama de la vida es una idea milenaria que ha sido utilizada a lo largo de la historia de la humanidad por místicos, filósofos y poetas para expresar su visión del trenzado y la interconexión entre todos los fenómenos naturales, humanos y sociales. 
La trama de la vida está constituida por redes dentro de redes. En cada escala y bajo un escrutinio más cercano, los nodos de una red se revelan como redes más pequeñas. Tendemos a organizar estos sistemas, todos ellos anidando en sistemas mayores, en un esquema jerárquico situando los mayores por encima de los menores a modo de pirámide invertida, pero esto no es más que una proyección humana. En la naturaleza no hay un "arriba" ni un "abajo" ni se dan jerarquías. Solo hay redes dentro de redes (Capra, 2010, p. 54).

Estas relaciones preferenciales pueden darse entre procesos o en el interior de un proceso determinado, lo cual da lugar a configuraciones con diversos grados de complejidad, que pueden ser estudiados a partir de una investigación fenomenológica.

\section{La fenomenología}

La fenomenología es un modelo epistémico derivado del fenomenismo, que a su vez es generado por el positivismo, y este emana del empirismo, que fue generado por el realismo, que es el modelo originario.

La fenomenología (Quillet, 1971) constituye "el estudio de los fenómenos, entendidos estos independientemente de la realidad de las que son manifestaciones. Se ha entendido también por fenomenología el estudio de los fenómenos psíquicos en sí mismos, tal como estos se dan diferenciando así la psicología de la lógica, que sería ciencia normativa del espíritu" (Citado en Fernández, 2007, p. 175).

Según Fernández (2007), en la filosofía contemporánea

[1]a fenomenología es la orientación, ante todo metodológica, iniciada por Husserl (1859-1938; Prossnitz-Moravia), que se propone describir todo lo que se ofrece a la intuición, tal como se da, prescindiendo de todo supuesto, o sea, procediendo a una reducción fenomenológica que pone entre paréntesis cualquier afirmación acerca de la realidad o irrealidad de lo que se intuye, y también de la existencia o inexistencia de un mundo al que esas intuiciones corresponderían. La fenomenología consiste, así, en una suspensión del juicio, es decir, en la abstención de toda afirmación o negación con respecto a la realidad de lo intuido (p. 58).

Husserl desarrolla la fenomenología a comienzos del siglo $X X$, afirmando que todo, incluso la naturaleza, es producto de la conciencia humana, por cuanto a través de nuestra experiencia es que podemos percibir y nombrar al mundo que nos rodea. La fenomenología es para Husserl el camino más apropiado para transformar la filosofía en ciencia estricta, en vista de la crisis general de la ciencia ocasionada por la pretensión del positivismo de reducir a científico todo conocimiento.

La elaboración de su crítica conduce a Husserl a la proposición de una lógica pura independiente de toda experiencia. La tarea de dicha lógica consiste en encontrar los conceptos y leyes que componen los elementos ideales de una teoría. En la actitud teórica, la naturaleza está ahí para que el sujeto la conozca, pero su conocimiento es insuficiente en la pretensión de conocer el mundo, pues ella no incluye construcciones éticas o estéticas (valores, obras de arte) que son también objetos de conocimiento y ciencia. 
En esta actitud la clave está en la forma como las vivencias de conciencia objetivantes (dóxicos) son ejecutadas en función de conocer. El conocimiento de algo no se da solo por la representación que el sujeto se hace, sino por la forma fenomenológica como el sujeto vive estos actos. No es tanto ver o percibir algo adoptando una actitud pasiva, es por el contrario vivir, actuar en cuanto yo, en sentido espacial, creyendo, juzgando, estar dirigido a lo objetivo con mirada activa.

En la fenomenología de Husserl (2011) se revive el término epojé con distinto sentido del término clásico. El mundo natural no queda negado ni se duda de su existencia. De esta manera, la epojé fenomenológica no es comparable ni a la duda cartesiana, ni a la suspensión escéptica del juicio, ni a la negación de la realidad por algunos sofistas, ni a la abstención de explicaciones propugnada, en nombre de una actitud libre de teorías y supuestos metafísicos, por el positivismo de Comte. Solo así es posible, según Husserl, constituir la conciencia pura o trascendental como residuo fenomenológico.

En la época actual, la fenomenología es considerada un método y a la vez una manera de ver el mundo. Esta doctrina se opone al psicologismo por su pretensión de reducir las leyes de la lógica a leyes inductivas de los procesos psíquicos.

Desde una mirada epistemológica, reducción (del latín reductio) significa distanciarse de la experiencia subjetiva, mediante la interrupción de las creencias sobre lo que está siendo vivenciado. De esta manera, la experiencia se muestra más viva y podemos reflexionar de manera sistémica, lo cual permite considerar y aquilatar en su justa medida la unidad de las cosas, es decir, el holos gestáltico.

\section{Conclusiones}

En efecto, estamos inmersos en una revolución científica que no solo abarca cambios en el ámbito de las ciencias físico-naturales, sino que además provoca metamorfosis en la forma en que comprendemos al ser humano y sus relaciones. Estamos inmersos en profundos cambios que se avecinan en la interpretación y configuración del pensamiento humano y en la comprensión de la sociedad, lo cual contribuirá al desarrollo de una teoría social humanista y configuracional.

\section{Referencias}

1. Bateson, G. (2010/1972). Pasos hacia una ecología de la mente. Una aproximación revolucionaria a la autocomprensión del hombre. Buenos Aires: Lumen.

2. Bateson, G. (2011/1979). Espiritu y naturaleza. Buenos Aires: Amorrortu Editores. Tercera edición.

3. Bertalanffy, L. V. (1976). Teoría General de Sistemas. Madrid: Fondo de Cultura Económica.

4. Bertalanffy, L. V. (1978). Tendencias en la teoría general de sistemas. Madrid: Alianza Editorial.
5. Bertalanffy, L. V. (2007). Teoría general de los sistemas. Argentina: Fondo de cultura económica.

6. Capra, F. (1988). Sabiduría Insólita: conversaciones con personajes notables. Barcelona: Kairós.

7. Capra, F. (1991). Pertenecer al Universo: encuentros entre ciencia y espiritualidad. Barcelona: Kairós.

8. Capra, F. (2003). Las conexiones ocultas. Implicaciones sociales, medioambientales, económicas y biológicas de una nueva visión del mundo. Barcelona: Anagrama. 
9. Capra, F. (2007). El tao de la física. Una exploración de los paralelismos entre la física moderna y el misticismo oriental. Málaga: Sirio.

10. Capra, F. (2008a). El punto crucial. Ciencia, sociedad y cultura naciente. Buenos Aires: estaciones.

11. Capra, F. (2008b). La ciencia de Leonardo. La naturaleza profunda de la mente del gran genio del renacimiento. Barcelona: Anagrama.

12. Capra, F. (2009). Sabiduría insólita. Conversaciones con personajes notables. Barcelona: Kairós.

13. Capra, F. (2010). La trama de la vida. Una nueva perspectiva de los sistemas vivos. Barcelona: Anagrama.

14. Dilthey, W. (1951). Ideas acerca de una psicología descriptiva y analítica. Obras completas. Vol. 6. México: FCE.

15. Fernández, I. O. (2007). Diccionario de investigación. Una comprensión holistica. Caracas: Ediciones Quirón.

16. Fuentes, H. (2009). El proceso de investigación cientifica desde la Teoría Holistico - Configuracional. Santiago de Cuba: CEES "Manuel F. Gran".

17. Fuentes, H., Álvarez, I. \& Matos, E. (2004). La teoría holístico - configuracional en los procesos sociales. Revista Pedagogía Universitaria, 9 (1).

18. González, F. (1997). Epistemología cualitativa y subjetividad. Ciudad de la Habana, Editorial Pueblo y Educación.

19. Grof, S. (s. f.). Psicología Transpersonal. Nacimiento, muerte $y$ trascendencia en psicoterapia. Barcelona: Kairós.
20. Husserl, E. (2011). La idea de la fenomenología. Barcelona: Herder.

21. Köhler, W. (1967). Psicología de la configuración. Introducción a los conceptos fundamentales. Madrid: Morata.

22. Laing, R. D. (1978). El yo dividido. México: F.C.E.

23. Laing, R. D. (1983). La voz de la experiencia. Barcelona: Crítica.

24. Laszlo, E. (1989). La gran bifurcación. Crisis y oportunidad: anticipación del nuevo paradigma que está tomando forma. Barcelona: Gedisa.

25. Ortiz, A. (2013). Configuralogia: paradigma epistemológico y metodológico en las ciencias humanas y sociales. Barranquilla: Antillas.

26. Ortiz, A. (2013). Epistemología e investigación configuracional en las Ciencias Humanas y Sociales. Fundamentos, eslabones, método y técnicas. Santa Marta: Inédito.

27. Prigogine, I. (2008). Las leyes del caos. Barcelona: Crítica.

28. Prigogine, I. (2009). ¿Tan solo una ilusión? Una exploración del caos al orden Barcelona: Tusquets.

29. Prigogine, I. (2012). El nacimiento del tiempo. Barcelona: Tusquets.

30. Quillet (1971). Diccionario Enciclopédico Quillet. Buenos Aires: Arístides. 